\title{
THE STRUCTURE OF INSEPARABLE FIELD EXTENTIONS
}

\author{
BY \\ WILLIAM C. WATERHOUSE( $\left.{ }^{1}\right)$
}

\begin{abstract}
The goal of this paper is to introduce some structural ideas into the hitherto chaotic subject of infinite inseparable field extensions. The basic discovery is that the theory is closely related to the well-developed study of primary abelian groups. This analogy undoubtedly has implications beyond those included here. We consider only modular extensions, which are the inseparable equivalent of galois extensions. $\$ 2$ and 3 develop the theory of pure independence, basic subfields, and tensor products of simple extensions. The following sections are devoted to Ulm invariants and their computation; the existence of nonzero invariants of arbitrary index is proved by means of a theorem which furnishes an actual connection between primary groups and inseparable fields. The final section displays some complications in the field extensions not occurring in abelian groups.
\end{abstract}

1. Preliminaries; modular extensions。

Theorem 1.l. Let $K$ and $\left\{L_{a}\right\}$ be subfields of some common field, and suppose $K$ is linearly disjoint from each $L_{a}$. Then $K$ is linearly disjoint from $L=\bigcap_{a_{a}}$

Proof. Suppose that there are $x_{1}, \ldots, x_{n}$ in $L$ linearly independent over $L \cap K$ but not over $K$. We may assume $n$ minimal; then there is a relation $\Sigma a_{i} x_{i}=0$ with $a_{i} \in K$ and $a_{1}=1$, and this relation is unique. The $x_{i}$ are in eaçh $L_{a}$ and are dependent over $K$, hence dependent over $K \cap L_{a^{*}}$ Since the dependence relation over $K$ is unique, the $a_{i}$ are in $K \cap L_{a^{*}}$ But this implies $a_{i} \in K \cap L$, which is impossible.

This theorem is basic and will be used repeatedly, usually without explicit citation.

Definition [5]. A purely inseparable field extension $K / k$ is modular if $K^{p^{n}}$ and $k$ are linearly disjoint, $n=1,2,3, \ldots$. (The concept is defined in [5] for more general extensions, but all the extensions we consider will be purely inseparable.)

If $K / k$ is a finite purely inseparable extension, then it is known

Received by the editors February 5, 1974.

AMS (MOS) subject classifications (1970). Primary 12F 15; Secondary 20K10.

(1) This work was supported in part by NSF Contract GP-25600. 
$\left[6\right.$, p. 433] that $K \otimes_{k} \bar{k}$ has the form $\bar{k}\left[X_{1}, \ldots, X_{n}\right] /\left(X_{1}^{q_{1}}, \ldots, X_{n}^{q_{n}}\right)$ where the $q_{i}$ are powers of the characteristic $p$; note for later reference that this truncated polynomial algebra is simply $\bar{k}[G]$ for $G$ a finite abelian $p$-group. Say that an extension $L$ splits $K$ if $K \otimes_{k} L \simeq L[G]$. Then there is a unique smallest splitting field $S(K / k)$; it contains $K$ and is itself a finite purely inseparable extension of $k$. Furthermore, $K$ is its own splitting field if and only if it is a tensor product of simple extensions [6, p. 425], and this condition is equivalent to modularity [5, p. 403]. Thus the finite modular extensions in this theory play a role like that of those finite separable extensions which are their own splitting fields, i.e. the galois extensions.

Proposition 1.2. Let $K / k$ be a purely inseparable extension, $\left\{L_{a}\right\}$ a family of intermediate fields.

(a) If each $L_{a}$ is modular over $k$, their intersection is modular over $k$.

(b) If $K$ is modular over each $L_{a^{\prime}}$ it is modular over their intersection.

(c) If the $L_{a}$ are directed by inclusion and each is modular over $k$, their union is modular over $k$.

(d) If the $L_{a}$ are directed by inclusion and $K$ is modular over each of them, it is modular over their union.

Proof. Since the pth power map is injective, we have $\left(\cap L_{\alpha}\right) p^{n}=$ $\bigcap\left(L p_{a}^{n}\right)$. Hence (a) as well as (b) follows from 1.1. Statements (c) and (d) are even easier, needing only the fact that linear disjointness is a condition of finite type.

Part of this proposition was proved in a different way in [3].

Proposition 1.3. Let $K / k$ be purely inseparable. The following are equivalent:

(a) $K / k$ is modular.

(b) $K$ is a directed union of finite modular extensions of $k$.

(c) For every finite extension $F / k$ contained in $K$, the splitting field $S(F / k)$ is contained in $K$.

Proof. Let $F$ be any finite purely inseparable extension of $k$, and consider the sequence $S(F / k), S(S(F / k) / k), \ldots$. By $\left[6, p_{0} 429\right]$ it stabilizes after finitely many steps, and the result is the smallest modular extension of $k$ containing $F$. Hence from (c) we can deduce that every finite subextension is contained in a finite modular extension, thus getting (b). We can deduce (a) from (b) by 1.2 (c). Finally, assume (a); let $F$ be a finite subextension, and let $L$ be the smallest modular extension containing it. We have $L \cap K$ modular over $k$ by 1.2 (a), and $F \subseteq L$, so by minimality $L=L \cap K$; thus $S(F / k) \subseteq L \subseteq K$. 
This proposition shows that modular extensions in general are the inseparable analogue of infinite galois extensions.

We must also put on record the familiar result about linear disjointness in towers [2, p. 162], which we use in the form derived by Sweedler [5, p. 206]:

Proposition 1.4. Let $L / k$ be purely inseparable, $K$ an intermediate field.

(a) The following are equivalent:

(i) $K$ and $k L^{p^{n}}$ are linearly disjoint over $k\left[K \cap L^{p^{n}}\right]$ for all (positive) $n$, and $L$ is modular over $k$.

(ii) $K \cap L^{p^{n}}$ and $k$ are linearly disjoint for all $n$, and $L$ is modular over $K$.

(b) The following are equivalent:

(i) $K^{p^{n}}$ and $L^{p^{n}} \cap k$ are linearly disjoint for all $n$, and $L$ is modular over $k$.

(ii) $L^{p^{n}}$ and $k K^{p^{n}}$ are linearly disjoint over $K^{p^{n}}\left[L^{p^{n}} \cap k\right]$ for all $n$, and $K$ is modular over $k$.

Remark 1.5. To illustrate how 1.1 and 1.4 are used, we insert here an argument which will arise later. Let $L / k$ be modular. Then $L^{p^{r}}$ and $k^{1 / p}$ are linearly disjoint, since $p$ th power is an isomorphism. Clearly $L^{p^{r}}$ and $L$ are linearly disjoint, so $L^{p^{r}}$ and $L \cap k^{1 / p}$ are linearly disjoint. That is, $L$ is modular over $L \cap k^{1 / p}$. Also, $\left(L \cap k^{1 / p}\right) \cap L^{p^{n}}$ equals $L^{p^{n}} \cap$ $k^{1 / p}$, and by 1.1 this is linearly disjoint from $k$. Thus by 1.4 (a) we conclude that $k L^{p^{n}}$ and $L \cap k^{1 / p}$ are linearly disjoint over $k\left[L^{p^{n}} \cap k^{1 / p}\right]$, and in particular have that field as their intersection.

2. Pure independence and basic subfields.

Definition. Let $K / k$ be modular. A subset $\left\{x_{i}\right\}$ of $K \backslash k$ is pure independent if for each $n$ the monomials $\Pi x_{i}^{e_{i}}$ with $0 \leq e_{i}<p^{n}$, almost all $e_{i}=0$, and $e_{i}<\left|k\left(x_{i}\right): k\right|$ are linearly independent over $k K^{p^{n}}$.

This definition says that no polynomial in the $x_{i}$ lies in $k K^{p^{n}}$ unless it obviously does; tensor product generators, for instance, must have this property. If $K^{p} \subseteq k$, pure independence reduces to the usual concept of $p$-independence [2, p. 180].

Definition. Let $K / k$ be purely inseparable. An intermediate field $F$ is called pure if $F$ and $k K^{p^{n}}$ are linearly disjoint over $k F^{p^{n}}$ for all $n$. Note that then $k\left[K^{p^{n}} \cap F\right]$ equals $k F^{p^{n}}$, and if $K / k$ is modular it follows by 1.4 (a) that $K / F$ is also modular.

Proposition 2.1. Let $K / k$ be modular. If $\left\{x_{i}\right\}$ is pure independent, 
then the subextension $F$ generated by the $x_{i}$ is pure and is the tensor product of the simple extensions $k\left(x_{i}\right)$. Conversely, if $F$ is a pure subfield of the form $\bigotimes_{k}\left(x_{i}\right)$, then the $x_{i}$ are pure independent.

Proof. The definition of pure independence shows that the monomials $\Pi x_{i}{ }_{i}{ }_{i}$ with $0 \leq e_{i}<\left|k\left(x_{i}\right): k\right|$ and almost all $e_{i}=0$ are independent over $k$; thus the surjection $\otimes k\left(x_{i}\right) \rightarrow F$ is also injective. We have then $k F^{p^{n}}=$ $\bigotimes_{k}\left(x_{i}^{p^{n}}\right)$, and a basis for $F$ over $k F^{p^{n}}$ is given by the monomials with $e_{i}<\left|k\left(x_{i}\right): k\right|$ and $0 \leq e_{i}<p^{n}$. By definition again these are still independent over $k K^{p^{n}}$, and thus $F$ is pure. The converse implication is equally easy.

Proposition 2.2. Let $K / k$ be modular, and assume $K \cap k^{1 / p}=k K^{p^{n}} \cap$ $k^{1 / p}$ for all $n$. Then $K$ is relatively perfect over $k$, i.e. $k K^{p}=K$.

Proof. We show inductively on $r$ that $K \cap k^{1 / p^{r}} \subseteq k K^{p^{n}}$ for all $n$; by hypothesis this is true for $r=1$. Assume it for $r$ and take $x \in K \cap k^{1 / p^{r+1}}$. Then $x^{p} \in K \cap k^{1 / p^{r}}$, so $x^{p} \in k K^{p^{n+1}}$ by induction. Say $x^{p}$ is a $k$-linear combination of elements $y_{i}^{p^{n+1}}$ with $y_{i} \in K$. Then $x^{p}$ and $\left(y_{i}^{p^{n}}\right)^{p}$ are in $K^{p}$, and $x^{p}$ is in the $k$-span of the others; by modularity it is in the $\left(K^{p} \cap k\right)$ span. Taking pth roots we get $x$ as a $\left(K \cap k^{1 / p}\right)$-linear combination of the $y_{i}^{p^{n}}$, and hence $x \in\left(K \cap k^{1 / p}\right) K^{p^{n}} \subseteq\left(k K^{p^{n}}\right) K^{p^{n}}=k K^{p^{n}}$.

Definition. Let $K / k$ be modular. A basic subfield of $K / k$ is an intermediate field $F$ such that

(i) $F$ is a tensor product of simple extensions of $k$,

(ii) $F$ is pure, and

(iii) $K$ is relatively perfect over $F$.

Theorem 2.3. Let $K / k$ be modular. Then the basic subfields are precisely the subextensions generated by maximal pure independent sets.

Proof. Let $F$ be generated by a maximal pure independent set $\left\{x_{i}\right\}$; in view of 2.1 the problem is only to prove $K$ relatively perfect over $F$. As remarked after the definition of purity, $K / F$ is automatically modular. If it is not relatively perfect, then 2.2 implies that we can find an $n$ (possibly $n=1$ ) such that $K \cap F^{1 / p}$ is contained in $F K^{p^{n-1}}$ but not in $F K^{p^{n}}$.

Remark 1.5 tells us that $K \cap F^{1 / p}$ and $F K^{p^{n-1}}$ have intersection $F\left[F^{1 / p} \cap K^{p^{n-1}}\right]$, and thus $K \cap F^{1 / p}$ is generated over $F$ by elements $x^{p^{n-1}}$ with $x \in K$. We can therefore choose such an $x$ with $x^{p^{n-1}} \notin F K^{p^{n}}$. By purity, $F \cap k K^{p^{n}}=k F^{p^{n}}$; hence $x^{p^{n}}$ is in $k F^{p^{n}}$, say a $k$-linear combination of $y_{j}^{p^{n}}$ with $y_{j} \in F$. As $k$ is linearly disjoint from $K^{p^{n}}$, we can take 
this combination with coefficients in $k \cap K^{p^{n}}$. Taking $p^{n}$ th roots we get

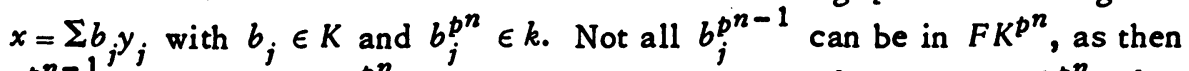
$x^{p^{n-1}}$ would be in $F K^{p^{n}}$. Thus we have an element $b$ in $K$ with $b^{p^{n}} \in k$ and $b^{p^{n-1}} \notin F K^{p^{n}}$. We now claim that $\left\{x_{i}\right\} \cup\{b\}$ is pure independent.

Suppose there is a relation among the properly restricted monomials $\Pi x_{i}^{e} i b^{e}$ over $k K^{p^{m}}$. All of them have $0 \leq e<p^{m}$ and $e<p^{n}=|k(b): k|$. Grouping the terms with the same $e$, we apparently have a relation among the $b^{e}$ over $F K^{p^{m}}$. If $m \leq n$ this means the degree of $b$ over $F K^{p^{m}}$ is less than $p^{m}$, and so $b^{p^{m-1}} \in F K^{p^{m}}$; but this is impossible, since raising to the $p^{n-m}$ th power would give $b^{p^{n-1}} \in F K^{p^{n}}$. For $m>n$ we similarly deduce degree less than $p^{n}$ and $b^{p^{n-1}} \in F K^{p^{m}} \subseteq F K^{p^{n}}$. Thus in either case we see that the coefficients of each power $b^{e}$ must be zero. But these now are relations among monomials in the $x_{i}$, and by pure independence all the coefficients in them must be zero. Thus we have only the trivial relation, and $\left\{x_{i}\right\}$ $U\{b\}$ is pure independent, contrary to the assumption of maximality.

Conversely, now, suppose $F=\bigotimes k\left(x_{i}\right)$ is a basic subfield; by 2.1 all we need to show is that $\left\{x_{i}\right\}$ is maximal. Suppose that $\left\{x_{i}\right\} \cup\{b\}$ is pure independent, with $|k(b): k|=p^{n}$. Then $b$ is in the span of $F$ over $k K^{p^{n}}$, since by relative perfection $K=F K^{p^{n}}$. But by purity $F(b)$ and $k K^{p^{n}}$ are linearly disjoint over $k F(b)^{p^{n}}=k F^{p^{n}}$, so $b$ is in $F\left[k F^{p^{n}}\right]=F$, which is clearly impossible.

\section{Corollary 2.4. Basic subfields exist.}

Proof. Pure independence is a condition of finite type, and so maximal pure independent sets exist.

Corollary 2.5 (Sweedler). Let $K / k$ be modular and $K^{p^{n}} \subseteq k$. Then $K$ is a tensor product of simple extensions.

Proof. Let $F$ be basic; then $K=F K^{p^{n}}=F$.

Remarks. (i) We shall see in 4.5 that in any maximal pure independent set the number of $x_{i}$ of each degree is an invariant. The actual field $F$, however, is far from unique. This can be shown easily by imitating the corresponding examples for groups, e.g. [1, p. I-147]; indeed, it can be deduced from those examples (see the Remark in \$6).

(ii) The process used in $[3, \$ 2]$ appears to be in essence a construction of basic subfields. The nonuniqueness was not noted there, and $[3$, Theorem 16] might be misleading for that reason.

Proposition 2.6. Let $K / k$ be modular, $B$ a pure modular subextension 
such that $B^{p^{n}} \subseteq k$ for some n. Then $B$ is a tensor factor of $K$ over $k$.

Proof. By 2.5 we know $B$ is a tensor product of simple extensions; let $X$ be a set of tensor generators of $B$ over $k$. By 2.1 the set $X$ is pure independent in $K$. Let $E=k K^{p^{n}}$; by purity $E$ is linearly disjoint from $B$ over $k B^{p^{n}}=k$. It follows that the elements of $X$ are pure independent over $E$. Extend $X$ to a maximal pure independent set $X \cup Y$ for $K$ over $E$; as $K^{p^{n}} \subseteq E$, we have $K=E(X \cup Y)$. Set $H=E(Y)$, so $B H=K$. Since $X \cup Y$ is pure independent over $E$, the basis of $B$ over $k$ given by monomials in the elements of $X$ is still independent over $E(Y)$. Thus $K=B \otimes_{k} H$.

The condition $B^{p^{n}} \subseteq k$ is necessary; indeed, a basic subfield need not be a tensor factor. Again examples to illustrate this can be deduced from those for groups, e.g. [1, p. I-139].

We conclude with a result needed in the next section. Like 2.2 , it demonstrates how $K \cap k^{1 / p}$ controls the structure of modular extensions.

Proposition 2.7. Let $K / k$ be modular, $E$ a pure subfield with $E \cap k^{1 / p}$ $=K \cap k^{1 / p}$. Then $E=K$.

Proof. Suppose inductively $E \cap k^{1 / p^{r}}=K \cap k^{1 / p^{r}}$, and take an $x \epsilon$ $K \cap k^{1 / p^{r+1}}$. Then $x^{p} \in E$, so by purity $x^{p} \in k E^{p}=E \cap k K^{p}$. Write it as a $k$-linear combination of $y_{i}^{p}$ with $y_{i} \in E$, so that $x$ is a $k^{1 / p-l i n e a r ~ c o m b i n a-~}$ tion of the $y_{i}$. Since $K / k$ is modular, $x$ is a linear combination of the $y_{i}$ over $K \cap k^{1 / p}=E \cap k^{1 / p}$; as the $y_{i}$ are in $E$, so is $x_{\text {. }}$

3. Tensor products of simple extensions.

Definition. Let $K / k$ be purely inseparable. An element in $K$ has height $<n$ if it is not in $k K^{p^{n}}$. An intermediate field $E$ has bounded height in $K / k$ if its elements not in $k$ have bounded height, i.e. $E \cap k K^{p^{n}}$ $=k$ for some $n$.

Theorem 3.1. Let $K / k$ be modular. The following are equivalent:

(i) $K$ is a tensor product of simple extensions.

(ii) $K$ can be written as an increasing union of intermediate fields $F_{1} \subseteq F_{2} \subseteq \cdots$ where each $F_{n}$ has bounded height in $K$ and $K / F_{n}$ is modular.

(iii) $K \cap k^{1 / p}$ can be written as an increasing union of intermediate fields $S_{1} \subseteq S_{2} \subseteq \cdots$ where each $S_{n}$ has bounded height in $K$ and $K / S_{n}$ is modular.

Proof. If $K$ is a tensor product of simple extensions, let $X$ be a corresponding pure independent set, and let $F_{n}$ be the intermediate field generated by the elements of $X$ with degree $\leq p^{n}$; then (ii) is satisfied. 
When (ii) holds, let $S_{n}=F_{n} \cap k^{1 / p}$. Clearly the $S_{n}$ have bounded height and exhaust $K \cap k^{1 / p}$; we know by 1.5 that $K$ is modular over $K \cap k^{1 / p}$, so by 1.2 (b) it is modular over $S_{n}$.

The problem then is to prove (i) assuming (iii). We do this by inductively constructing pure independent sets $X_{1} \subseteq X_{2} \subseteq \cdots$ such that $k\left(X_{n}\right) \cap k^{1 / p}=$ $S_{n}$; by 2.7 we will then have $K=k\left(U X_{n}\right)$. Suppose therefore that we already have $X_{n-1}$, and expand it to a pure independent set $X_{n}$ maximal among those for which $k\left(X_{n}\right) \cap k^{1 / p} \subseteq S_{n}$. The induction step will be complete if we can show that $k\left(X_{n}\right) \cap k^{1 / p}=S_{n}$.

Suppose the intersection is smaller. Then as $S_{n}$ has bounded height we can find an $r \geq 0$ such that $k K^{p^{r}} \cap S_{n} \notin k\left(X_{n}\right)$ but $k K^{p^{r+1}} \cap S_{n} \subseteq k\left(X_{n}\right)$. Since $K$ is modular over $S_{n}$ and over $k$, we deduce from $1.4(a)$ that $S_{n} n$ $k K^{p^{r}}=k\left[S_{n} \cap K^{p^{r}}\right]$, and hence there must be an element $b^{p^{r}}$ in $S_{n}$ not in $k\left(X_{n}\right)$. If we can now show $X_{n} \cup\{b\}$ is pure independent, we will be through. For then $k\left(X_{n} \cup\{b\}\right)=k(b) \otimes k\left(X_{n}\right)$, and the intersection of that with $k^{1 / p}$ is $k\left(b^{p^{r}}\right) \otimes\left[k\left(X_{n}\right) \cap k^{1 / p}\right] \subseteq S_{n}$, contrary to the maximality of $X_{n}$.

For brevity set $F=k\left(X_{n}\right)^{n}$ and $L=F \cap k^{1 / p} \subseteq S_{n}$. If $X, \cup\{b\}$ is not pure independent we get a nontrivial relation among properly restricted monomials $\Pi x_{i}^{e} i b^{e}$; as in the next-to-last paragraph of 2.3 we find $b^{p^{r}} \epsilon$ $F K^{p^{r+1}}$. Hence there is an element in $F K^{p^{r+1}} \cap S_{n}$ not in $F$. We can thus finish the proof by showing that $F K^{p^{r+1}} \cap S_{n} \subseteq F$.

We begin with the tower $k \subseteq k F^{p^{m}} \subseteq F$, for any $m$; by 1.4 (b) we deduce that $F^{p}$ and $k F^{p^{m+1}}$ are linearly disjoint over $k^{p} F^{p^{m+1}}\left[k \cap F^{p}\right]=$ $F^{p^{m+1}} L^{p}$. [For this we need $k F^{p^{m}}$ modular over $k$, which is clear here since $F$ is a tensor product of simple extensions - cf. 4.1.] It follows then that $F^{p}$ and $k K^{p^{m+1}}$ are linearly disjoint over $F^{p^{m+1}} L^{p}$. For suppose elements $f_{i}^{p}$ in $F^{p}$ are linearly dependent over $k K^{p^{m+1}}$; they are in $F$, so by the purity of $F$ they are linearly dependent over $k F^{p^{m+1}}$. By the previous step they are then linearly dependent over $F^{p^{m+1}} L^{p}$.

Now let $x$ be in $F K^{p^{m}} \cap k^{1 / p}$, say $x=\Sigma f_{i} a_{i}$ with. $f_{i} \in F$ and $a_{i} \epsilon$ $k K^{p^{m}}$. Then $x^{p}=\Sigma f_{i}^{p} a_{i}^{p}$. Both $x^{p}$ and $a_{i}^{p}$ are in $k K^{p^{m+1}}$, and $x^{p}$ is a linear combination of the $a_{i}^{p}$ over $F^{p}$, whence by linear disjointness it is so over $F^{p^{m+1}} L^{p}$. Taking $p$ th roots we get $x$ in $F^{p^{m}} L\left[k K^{p^{m}}\right]=L K^{p^{m}}$. Thus we have shown $F K^{p^{m}} \cap k^{1 / p}=L K^{p^{m}} \cap k^{1 / p}$, and in particular $F K^{p^{r+1}} \cap S_{n}=L K^{p^{r+1}} \cap S_{n}$.

Consider finally the tower $L \subseteq S_{n} \subseteq K$. By purity we know $K$ is modular over $F$, whence as we saw earlier $K$ is modular over $L=F \cap k^{1 / p}$. Therefore we have $S_{n} \cap K^{p^{m}}$ linearly disjoint from $L$ for all $m$. By as: sumption also $K / S_{n}$ is modular. Hence by 1.4 (a) we have $S_{n} \cap L K^{p^{r+1}}=$ 
$L\left[S_{n} \cap K^{p^{r+1}}\right]$. But by choice of $r$ we know $S_{n} \cap k K^{p r+1} \subseteq F$, so $S_{n} \cap$ $L K^{p^{r+1}} \subseteq F$. Consequently we have $S_{n} \cap F K^{p^{n}+1} \subseteq F$, as desired.

This theorem of course gives another proof of 2.5 .

4. Ulm invariants of modular extensions. Our first structural analysis, in $\$ 2$, led us to write a modular $K / k$ as $K / F / k$ with $K / F$ relatively perfect and $F / k$ a tensor product of simple extensions; the two steps are relatively uncomplicated, but the construction is not unique. In this section we will give a decomposition with the relatively perfect extension at the bottom; it is more elaborate but canonical, and will yield numerical invariants.

Definition. Let $K / k$ be purely inseparable. For $\alpha$ an ordinal, the intermediate field $(K / k)^{\alpha}$ [or $k K^{p}{ }^{a}$ is constructed inductively as follows:

(i) $(K / k)^{0}=K$,

(ii) $(K / k)^{\dot{\alpha}+1}=k\left[(K / k)^{a}\right]^{p}$, and

(iii) $(K / k)^{\alpha}=\bigcap\left\{(K / k)^{\beta} \mid \beta<a\right\}$ for $a$ a limit ordinal. Write $(K / k)^{\infty}$ for $\bigcap(K / k)^{a}$. The first $\alpha$ for which $(K / k)^{a}=(K / k)^{\infty}$ is the length of $K$ over $k$.

We thus have a descending chain of intermediate fields. The finite stages $(K / k)^{n}=k K^{p^{n}}$ are of course familiar, but it seems to have been unconsciously supposed that the chain would always stabilize at $(K / k)^{\omega}=$ $\bigcap(K / k)^{n}$. Indeed, this is tacitly assumed in [3, Proposition 9], and I believe it is true for every inseparable extension in the literature. It may therefore be advisable to give at once an example where it is false. Let $K=$ $F_{p}\left(y, x_{1}, x_{2}, x_{3}, \ldots\right)$ where $y$ and the $x_{i}$ are indeterminates, and let $k$ be $\mathbf{F}_{p}\left(y^{p}, x_{1}^{p} / y, x_{2}^{p^{2}} / y, x_{3}^{p^{3}} / y, \cdots\right)$. Then $(K / k)^{\omega}=k(y)$ and $(K / k)^{\omega+1}=k$, and thus $K / k$ has length $\omega+1$. It is in fact true, as we will prove in 6.2 , that there are extensions of arbitrary prescribed length.

The definition in $\$ 3$ can be extended transfinitely: the elements in $K$ of height $a$ are those in $(K / k)^{\alpha}$ but not in $(K / k)^{a+1}$. The elements of $(K / k)^{\infty}$ must of course be assigned height $\propto_{\text {. }}$

Proposition 4.1. Let $K / k$ be modular. Then for every $a$,

(a) $(K / k)^{a}$ is modular over $k$, and

(b) $K$ is modular over $(K / k)^{\alpha}$.

Proof. We first prove (a) by induction on $\alpha$. If $\alpha$ is a limit ordinal, $(K / k)^{a}$ is an intersection of extensions already known to be modular, and so it is itself modular. Suppose now that $E=(K / k)^{a}$ is modular over $k$. By 1.3 we can write $E$ as a directed union $U E_{\lambda}$ where the $E_{\lambda}$ are finite modular extensions of $k$. Then $k E^{p}=\bigcup_{k E_{\lambda}^{p}}$, and the $k E_{\lambda}^{p}$ are modular 
over $k$ by computation since $E_{\lambda}$ is a tensor product of simple extensions. Thus $(K / k)^{a+1}=k E^{p}$ is modular over $k$.

For (b) we note that by 1.4 (a) we have $K$ modular over $(K / k)^{\alpha}$ if and only if $(K / k)^{a}$ and $k K^{p^{n}}$ are linearly disjoint over $k\left[(K / k)^{a} \cap K^{p^{n}}\right]$ for all $n$. If $\alpha$ is a limit ordinal, the modularity holds by induction and 1.2 (b). Also, the second condition is trivial for $n \geq a$, as there $(K / k)^{a} \cap K^{p^{n}}=$ $K^{p^{n}}$. Thus assuming the result for $a$ we must prove $(K / k)^{a+1}=$ $k\left[(K / k)^{a+1} \cap K^{p^{n}}\right]$ for $1 \leq n<a+1$. But we have

$$
\begin{aligned}
k\left[(K / k)^{a+1} \cap K^{p^{n}}\right] & \supseteq k\left[\left((K / k)^{a}\right)^{p} \cap K^{p^{n}}\right]=k\left[(K / k)^{a} \cap K^{p^{n-1}}\right]^{p} \\
& =k k^{p}\left[(K / k)^{a} \cap K^{p^{n-1}}\right]^{p}=k\left((K / k)^{a}\right)^{p}=(K / k)^{a+1} .
\end{aligned}
$$

Definition. Let $K / k$ be modular. The $a$ th Ulm invariant of the extension, $f_{a}(K / k)$, is the $p$-rank of $(K / k)^{a} \cap k^{1 / p}$ over $(K / k)^{a+1} \cap k^{1 / p}$.

Proposition 4.2. Let $K / k$ be modular.

(a) $(K / k)^{\infty}$ is relatively perfect over $k$, and is the largest relatively perfect subextension of $K$.

(b) If $f_{n}(K / k)=0$ for all finite $n$, i.e. if all elements of $K \cap k^{1 / p}$ have height $\geq \omega$, then $K$ is relatively perfect over $k$.

(c) If for some a one has $f_{a+n}(K / k)=0$ for all finite $n$, then the length of $K / k$ is $\leq a$

(d) If $K$ is a tensor product of simple extensions, then $K / k$ has length $\leq \omega$, and $f_{n}(K / k)$ equals the number of tensor generators of degree $p^{n+1}$.

Proof. Statement (a) is obvious, (d) is a straightforward computation, and (b) is a paraphrase of 2.2. Then (c) follows by applying (b) to $(K / k)^{a}$, which is modular over $k$ by 4.1 .

Proposition 4.3. Let $K / k$ be modular. Let $n$ and $r$ be finite with $r \geq 2$. Then

(a) $f_{n}(K / k)=f_{n}\left(K \cap k^{\left.1 / p^{n+r} / k\right)}\right.$ and

(b) $f_{n}(K / k)=f_{n}\left(K /(K / k)^{n+r}\right)$.

Proof. By 1.5 we have $k^{1 / p} \cap k K^{p^{s}}=k\left[k^{1 / p} \cap K^{p^{s}}\right]$. But obviously for $n+r>s$ we have $k^{1 / p} \cap K^{p^{s}}=k^{1 / p} \cap\left(K \cap k^{1 / p^{n+r}}\right)^{p^{s}}$. Thus the same fields occur in computing both sides of (a).

Write $L=k K^{p^{n}}$; then (b) says that

$$
\left|k^{1 / p} \cap L: k^{1 / p} \cap k L^{p}\right|=\left|\left(k L^{p^{r}}\right)^{1 / p} \cap L:\left(k L^{p^{r}}\right)^{1 / p} \cap k L^{p}\right| \text {. }
$$


Thus it suffices to show that $k^{1 / p} \cap L$ and $\left(k L^{p^{r}}\right)^{1 / p} \cap k L^{p}$ are linearly disjoint with intersection $k^{1 / p} \cap k L^{p}$ and join $\left(k L^{p^{r}}\right)^{1 / p} \cap L$. Obviously the intersection is right. We have $k L^{p}$ and $k^{1 / p}$ linearly disjoint since the former is modular over $k$; similarly $L$ and $\left(k L^{p^{r}}\right)^{1 / p}$ are linearly disjoint. Hence by 1.1 we have the necessary linear disjointness.

To show the join is as large as claimed, we write $L$ as a directed union of finite modular extensions; if the join is large enough for each of them, it is so for their union. Thus we may assume $L / k$ finite. Write it as a tensor product of $k\left(x_{i}\right)$ with $x_{i}$ of exponent $e_{i}$. Then the elements with $p$ th power in $k L^{p^{r}}$ are generated by $x_{i}^{d_{i}}$ where $d_{i}=p_{i}^{e_{i}-1}$ if $e_{i}<r$ and $d_{i}=p^{r-1}$ if $e_{i} \geq r$. The last type are in $\left(k L^{p^{r}}\right)^{1 / p^{2}} \cap k L^{p}$, and the others are in $k^{1 / p} \cap L$.

Lemma 4.4. Let $K / k$ be modular, $B$ a pure modular subextension over which $K$ is relatively perfect. Then the Ulm invariants of $K$ over $k K^{p^{n}}$ equal those of $B$ over $k B^{p^{n}}$.

Proof. By hypothesis $B$ and $k K^{p^{n}}$ are linearly disjoint with intersection $k B^{p^{n}}$ and join $K$. Thus a pure independent set giving tensor generators for $B$ over $k B^{p^{n}}$ also gives tensor generators for $K$ over $k K^{p^{n}}$.

Theorem 4.5. Let $K / k$ be modular. Let $X$ be any maximal pure independent set. Then the number of elements of $X$ having degree $p^{n+1}$ is $f_{n}(K / k)$, and thus is independent of the choice of $X$.

Proof. By 2.3 we know $B=k(X)$ is a basic subfield, and by 4.2 (d) the number of elements involved is $f_{n}(B / k)$. But $f_{n}(B / k)=f_{n}\left(B / k B^{p^{n+2}}\right)=$ $f_{n}\left(K / k K^{p^{n+2}}\right)=f_{n}(K / k)$.

5. Ulm invariants and group algebras. In this section we will compute the invariants of $\$ 4$ by a process which explicitly connects them with the usual Ulm invariants for groups. Recall [1, p. I-154] that if $G$ is a $p$ primary abelian group, the subgroups $p^{a} G$ are defined inductively by $p^{a+1} G=p\left(p^{a} G\right)$, starting with $p^{0} G=G$ and setting $p^{a} G=\bigcap\left\{p^{\beta} G \mid \beta<\alpha\right\}$ for $\alpha$ a limit ordinal. If $G[p]$ denotes $\{g \in G \mid p g=0\}$, then the groups $p^{\alpha} G \cap G[p]$ are vector spaces over $F_{p}$, and (in Kaplansky's terminology) the codimension of $p^{a+1} G \cap G[p]$ in $p^{a} G \cap G[p]$ is the ath Ulm invariant of $G$, denoted $f_{a}(G)$.

Let $k$ now be a field of characteristic $p$, and $A$ a commutative $k$ algebra; we can define $(A / k)^{\alpha}$ inductively just as for field extensions. If $L$ is a larger field, it is easy to show inductively that $\left(A \otimes_{k} L / L\right)^{a}=$ 
$(A / k)^{a} \otimes_{k} L$. If $A=k[G]$, moreover, a simple induction using the standard basis shows that $(A / k)^{a}=k\left[p^{a} G\right]$.

With the proper tool, the Ulm invariants of $G$ can now be recovered from $k[G]$. If $A$ is any (commutative) local $k$-algebra with residue field $k$, let $M$ be its maximal ideal and $J$ the ideal $\left\{a \in A \mid a^{p}=0\right\}$. We define the Ulm space $\mathcal{U}(A)$ to be $J / M J$. Clearly $A \mapsto \mathcal{U}(A)$ is a functor from such algebras to vector spaces over $k$.

Proposition 5.1. Let $G$ be a p-primary abelian group, $k$ a field of characteristic $p$. Then there is a natural isomorphism

$$
G[p] \otimes_{\mathrm{F}_{p}} k \simeq \mathrm{U}(k[G]) .
$$

Proof. We show first that $J$ is generated by the elements $g-1$ for $g \in G[p]$; it is enough to show this for $G$ finite, since any $G$ is a directed union of finite subgroups. If $\left\{g_{i}\right\}$ are basic generators for a finite $G$, then $k[G]$ has the form $k\left[X_{1}, \ldots, X_{n}\right] /\left(X_{1}^{q_{1}}, \ldots, X_{n}^{q_{n}}\right)$, where $X_{i}=g_{i}-1$ and $g_{i}$ has order $q_{i}=p^{e_{i}}$. Clearly two different monomials here have equal $p$ th powers only if both collapse to zero, and the pth power of a monomial is zero only if it involves a factor $X_{i}^{q_{i} / p}=g_{i}^{q_{i} / p}-1$. This yields the desired statement.

We thus have a natural map $G[p] \rightarrow \mathcal{U}$ sending $g$ to the class of $g-1$. If $g$ and $h$ are in $G[p]$, then $g h-1=(g-1)+(h-1)+(g-1)(b-1)$ and the last term is in $M J$; thus $G[p] \rightarrow \mathcal{U}$ is $F_{p}$-linear. Any multiple of $(g-1)$ has the form $($ scalar $)(g-1)+$ (element of $M)(g-1)$, so the image of $G[p]$ spans $U$ over $k$.

When $G$ is finite, the map from $G[p] \otimes k$ is injective as well as surjective; this is clear from direct inspection of $k[G]$, as above. If then $G_{1}$ $\subseteq G_{2}$ are finite, $G_{1}[p]$ injects into $G_{2}[p]$, and hence accordingly $U\left(k\left[G_{1}\right]\right)$ injects into $\mathcal{U}\left(k\left[G_{2}\right]\right)$. Since $\mathcal{U}(k[G])$ in general is the direct limit of the Ulm spaces of finite subgroup algebras, it follows that $G[p] \otimes k$ always injects into $\mathrm{U}(k[G])$.

Corollary 5.2. Each $\mathrm{U}\left((k[G] / k)^{a}\right)$ injects into $\mathrm{U}(k[G])$, and the codimension of $\mathrm{U}\left((k[G] / k)^{a+1}\right)$ in $\mathrm{U}\left((k[G] / k)^{a}\right)$ is $f_{a}(G)$.

In particular, of course, the Ulm invariants of $G$ are determined by the algebra $k[G]$; a less transparent proof of this fact was given in [4]. We may note that $\operatorname{dim}_{\mathrm{F}_{p}} G[p] \cap p^{\propto} G$, which is the number of $\mathrm{Z}\left[p^{\propto}\right]$ summands in $G$, is similarly determinable from the Ulm space of $k[G]$.

For arbitrary algebras $\mathrm{U}(A)$ may be zero, or the $\mathrm{U}\left((A / k)^{\alpha}\right)$ may not 
inject into $\mathcal{U}(A)$. When they do, however, it is natural to call the codimensions in 5.2 the Ulm invariants of $A$. The Ulm invariants of $\$ 4$ can be recovered by precisely this process, as we now show.

Theorem 5.3. Let $K / k$ be modular. Let $L$ be the perfect closure of $k$, and let $A$ be the L-algebra $K \otimes_{k} L$. There is a natural map from $K \cap$ $k^{1 / p}$ to $U(A)$ sending $x$ to the class of $x \otimes 1-1 \otimes x$. Under this map a set $\left\{x_{i}\right\}$ goes to a basis (resp. an independent set, a spanning set) if and only if $\left\{x_{i}\right\}$ is a p-basis (resp. a p-independent set, a generating set) for $K \cap k^{1 / p}$ over $k$.

Proof. Suppose first that $K / k$ is finite and $K \subseteq k^{1 / p}$; let $\left\{x_{i}\right\}$ be a

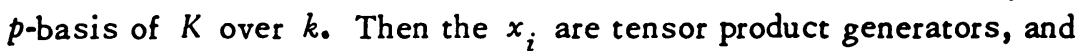
$K \otimes L$ is $\otimes L\left[X_{i}\right] / X_{i}^{p}$ where $X_{i}=x_{i} \otimes 1-1 \otimes x_{i}$. Clearly then the images of the $x_{i}$ are a basis for $\mathcal{U}(K \otimes L)$. Passing to a direct limit over finite subextensions we see that the same result holds for infinite $K \subseteq k^{1 / p}$. Furthermore, any $p$-independent set can be expanded to a $p$-basis, and so must go to an independent set; similarly any generating set contains a $p$ basis, and hence goes to a spanning set. In addition, if $F$ is an intermediate field, then a $p$-basis of $F$ extends to one of $K$, and thus $U(F \otimes L)$ injects into $U(K \otimes L)$.

Still assuming $K \subseteq k^{1 / p}$, let $\left\{x_{i}\right\}$ be elements whose images are independent. If the $x_{i}$ are not $p$-independent, some one of the $x_{1}$ is in the field $F$ generated over $k$ by the others. The images of $x_{i}$ for $i \neq 1$ then span $U(F \otimes L)$, so the image of $x_{1}$ is dependent on them there and hence is dependent on them in $U(K \otimes L)$. This is impossible, and so the $x_{i}$ are $p$-independent. If on the other hand $\left\{x_{i}\right\}$ is a set of elements whose images span $U(K \otimes L)$, let $F$ be the subextension they generate. If $F$ is not all of $K$ we can choose a $p$-basis of $F$ and extend it to a $p$-basis of $K$; we get thereby an element in $U(K \otimes L)$ not in $U(F \otimes L)$, which is impossible since $U(F \otimes L)$ contains the images of the $x_{i}$. Thus the theorem is completely proved for the case $K \subseteq k^{1 / p}$.

Now let $K / k$ be arbitrary finite modular with tensor product generators $y_{i}$ having degrees $q_{i}=p^{e_{i}}$. Then $K \otimes L$ is $\otimes L\left[Y_{i}\right] /\left(Y_{i}^{q_{i}}\right)$ where $Y_{i}=$ $y_{i} \otimes 1-1 \otimes y_{i}$. The computation in 5.1 shows that the elements $Y_{i}^{q_{i} / p}$ give a basis for $\mathcal{U}(K \otimes L)$. But the elements $y_{i}^{q_{i} / p}$ are a $p$-basis for $K \cap$ $k^{1 / p}$, and hence go to a basis for $\mathcal{U}\left(\left(K \cap k^{1 / p}\right) \otimes L\right)$. Since a basis thus goes to a basis, the map $U\left(\left(K \cap k^{1 / p}\right) \otimes L\right) \rightarrow \mathcal{U}(K \otimes L)$ is an isomorphism. Writing any modular $K / k$ as a direct limit of finite modular extensions, we see that the map is still an isomorphism there. Hence the theorem is reduced to $K \cap k^{1 / p}$, where we have proved it. 
Porism 5.4. If $F$ is an intermediate field modular over $k$, then $\mathrm{U}(F \otimes L)$ $\rightarrow \mathcal{U}(K \otimes L)$ is an injection.

Corollary 5.5. If $K / k$ is modular, then the Ulm invariants of $K / k$ are the same as the Ulm invariants of the algebra $K \otimes L$.

Proof. The p-rank of $(K / k)^{a} \cap k^{1 / p}$ over $(K / k)^{a+1} \cap k^{1 / p}$ equals the number of elements added to a $p$-basis of the latter over $k$ to get a $p$-basis of the former over $k$. Thus it equals the codimension of $\mathrm{U}\left((K / k)^{a+1} \otimes L\right)$ in $U\left((K / k)^{\alpha} \otimes L\right)$. The p-rank is $f_{\alpha}(K / k)$; the codimension is the Ulm invariant of $K \otimes L$, since $(K / k)^{a} \otimes L=(K \otimes L / L)^{a}$.

This need not be true, with the definition of $\$ 4$, if $K / k$ is not modular. If one wants to define Ulm invariants for nonmodular extensions, they should probably be the invariants of $K \otimes L$.

Corollary 5.6. If $K / k$ is modular and is a form of a group algebra, i.e. $K \otimes L$ is isomorphic to a group algebra $L[G]$ then $f_{a}(K / k)=f_{a}(G)$.

Theorem 5.7. Let $K / k$ be modular, $L$ the perfect closure of $k$, and a a limit ordinal. Then

$$
\mathcal{U}\left((K / k)^{\alpha} \otimes L\right)=\bigcap\left\{U\left((K / k)^{\beta} \otimes L\right) \mid \beta<\alpha\right\} .
$$

Proof. We of course use 5.4 to identify these spaces with subspaces of $\mathcal{U}(K \otimes L)$. The inclusion $\subseteq$ is obvious. Take then an element $v$ in the right-hand side. It can be expressed using finitely many elements from $K \cap k^{1 / p}$; among such expressions choose one using the smallest number of elements not in $(K / K)^{a} \cap k^{1 / p}$. Let $M$ be the intermediate field generated by these elements. We clearly must have $M$ linearly disjoint from $(K / k)^{a}$ $\cap k^{1 / p}$ over $k$, as otherwise we could drop one of the generators of $M$ and still span the same space together with $\mathcal{U}\left((K / k)^{a} \otimes L\right)$.

Lemma 5.8. Let $K / k$ be algebraic. Let $\left\{E_{\beta}\right\}$ be a family of intermediate fields directed by inverse inclusion, and let $E=\bigcap_{\beta}$. Suppose $M$ is an intermediate field linearly disjoint from $E$ with $|M E: E|$ finite. Then $M$ is linearly disjoint from all sufficiently small $E_{\beta}$.

Proof. Let $B$ be a linear basis for $M$ over $M \cap E$; it is then a basis for $M E$ over $E$, and so is finite. Choose any $\beta$; then we choose a subset $B_{\beta}$ of $B$ which is a basis for $M E_{\beta}$ over $E_{\beta}$. Consider some $E_{\gamma} \subseteq E_{\beta}$; if $B_{\beta}$ is no longer a basis of $M E_{\gamma}$ over $E_{\gamma}$ it can be expanded to a larger subset $B_{\gamma}$ which is. Since $B$ is finite, successive expansions in this way must eventually stop; thus we get an $E_{\gamma}$ and a subset $B_{\gamma}$ of $B$ 
such that $B_{\gamma}$ is a basis of $M E_{\delta}$ over $E_{\delta}$ whenever $E_{\delta} \subseteq E_{\gamma^{*}}$ Obviously it is enough now to show $B_{\gamma}=B$. Suppose therefore that there is some $b$ in $B \backslash B_{\gamma^{*}}$ We can write $b=\Sigma a_{i} b_{i}$ uniquely with $b_{i} \in B_{\gamma}$ and $a_{i} \in E_{\gamma^{*}}$ The same is true for each smaller $E_{\delta}$, so the $a_{i}$ are in $\bigcap\left\{E_{\delta} \mid E_{\delta} \subseteq E_{\gamma}\right\}$. But that intersection is $E$, since the original intersection was directed; and thus we have a contradiction.

Applying this lemma in our previous situation, we see that $M$ is linearly disjoint from all sufficiently small $(K / k)^{\beta} \cap k^{1 / p}$. Since $M$ is finite, the intersection of the two will also be $k$ eventually. But now we can choose a $p$-basis $X_{1}$ of $(K / k)^{a} \cap k^{1 / p}$, expand it to a $p$-basis $X_{1} \cup X_{2}$ of $(K / k)^{\beta} \cap k^{1 / p}$, and add in a $p$-basis $X_{3}$ of $M$ while still staying independent. In $U(K \otimes L)$ the images $Y_{1} \cup Y_{2} \cup Y_{3}$ will then be linearly independent. The set $Y_{1} \cup Y_{2}$ spans $U\left((K / k)^{\beta} \otimes L\right)$, so $v$ is in its span; and by definition of $M$ we have $v$ in the span of $Y_{1} \cup Y_{3}$. But that implies $v$ is in the span of $Y_{1}$, i.e. $v$ is in $\mathrm{U}\left((K / k)^{a} \otimes L\right)$.

This is a nontrivial property which $K \otimes L$ shares with group algebras; there are other algebras for which it is false.

6. An existence theorem.

Theorem 6.1. Let $G$ be a p-primary abelian group. Then there is a modular extension $K / k$ which is a form of $k[G]$, in fact for which $K \otimes_{k} K$ $\simeq K[G]$

Proof. Let $F$ be a field of characteristic $p$. Let $0 \rightarrow R \rightarrow P \rightarrow G$ $\rightarrow 0$ be a resolution of $G$ with $P$ and hence $R$ free abelian groups. Then $F[P]$ is equal to $F\left[\left\{X_{a}, 1 / X_{a}\right\}\right]$ where $X_{a}$ runs over a basis of $P$; in particular it is an integral domain, and $F[R]$ is a subdomain. Let $K$ and $k$ be their fraction fields. It is easy to verify that $X_{a} \otimes X_{\beta} \mapsto \bar{X}_{\alpha} \otimes X_{a} X_{\beta}$ defines an isomorphism

$$
F[P] \otimes_{F[R]} F[P] \simeq F[G] \otimes_{F} F[P] .
$$

Now since $G$ is torsion, $F[P]$ is integral over $F[R]$, and hence $K=$ $\left.F[P] \otimes_{F[R}\right]^{k}$. Tensoring the above isomorphism with $k$ then yields

$$
K \otimes_{k} K \simeq F[G] \otimes_{F} K=K[G] .
$$

It now follows automatically that $K / k$ is modular. For if $E$ is a finite subextension, $E \otimes_{k} K$ is isomorphic to a subalgebra of $K\left[G_{0}\right]$ for some finite subgroup $G_{0}$ of $G$. It follows $\left[6, \mathrm{p}_{0} 423\right]$ that $E \otimes_{k} K$ is split, and so $K$ contains the splitting field $S(E / k)$. Hence $K / k$ is modular by 1.3 . 
Corollary 6.2. Let $\lambda$ be any ordinal. Then there is a modular $K / k$ with $(K / k)^{\lambda}=k$ and length precisely $\lambda$.

Proof. It is known [1, pp. II-57 and II-210] that there is a group $G$ with $p^{\lambda} G=0$ and $p^{\beta} G \neq 0$ for $\beta<\lambda$. Take $K / k$ in the theorem corresponding to this $G$, noting that we already know $(K / k)^{\beta} \otimes K=\left(K[G 1 / K)^{\beta}=K\left[p^{\beta} G\right]\right.$.

Corollary 6.3. If $K / k$ is a countable-dimensional modular extension, then $\alpha \mapsto f_{a}(K / k)$ is a function from countable ordinals to cardinals $\leq \boldsymbol{N}_{0}$ with the following properties:

(i) it is identically zero from some point on, and

(ii) it nowhere has an infinite string of successive zero values followed by a nonzero value.

Conversely, any such function arises from some countable-dimensional modular extension.

Proof. Condition (ii) is $4.2(c)$. Since $U(K \otimes L)$ is countable-dimensional, clearly all $f_{a}$ are $\leq \boldsymbol{x}_{0}$; also there cannot be an uncountable well-ordered chain of subspaces, so (i) holds. The converse follows from combining 6.1, 5.6 , and the corresponding existence theorem for countable groups [1, p. II$65]$, since in 6.1 we clearly have $(K: k)=\#(G)$.

Remark. In the construction of 6.1 any subgroup of $G$ will correspond to an intermediate modular field (though of course there will be many other intermediate fields also). Examples of subgroups with certain properties can often therefore be carried over to examples on fields. For instance, there are well-known examples showing that one basic subgroup may be properly contained in an other, and that a pure subgroup of unbounded height need not be a direct summand; as mentioned in $\$ 2$, these yield corresponding examples for fields.

7. Complications and counterexamples. In this section we show that two analogues of abelian group theorems are false for fields. The first corresponds to the famous theorem of Prüfer $[1, \mathrm{p} . \mathrm{I}-88]$ that countable primary groups with no elements of infinite height are direct sums of cyclic groups.

Proposition 7.1. There is a countable-dimensional modular extension $K / k$ with $(K / k)^{\omega}=k$ which is not a tensor product of simple extensions.

Proof. Let $k=\mathrm{F}_{p}\left(b, a_{0}, a_{1}, a_{2}, a_{3}, \ldots\right)$ where $b$ and the $a_{i}$ are indeterminates. Let $y_{0}=b^{1 / p}$ and $x_{n}=a_{n}^{1 / p}$. Define inductively $y_{n}=$ $y_{n-1}+x_{n-1} x_{n}$ for $n=1,2,3, \ldots$. Let 


$$
K_{n}=k\left(y_{n}^{1 / p^{n}}, x_{0}, x_{1}^{1 / p}, x_{2}^{1 / p^{2}}, x_{3}^{1 / p^{3}}, \ldots, x_{n}^{1 / p^{n}}\right)
$$

Since $x_{0}, x_{1}, x_{2}, \ldots, x_{n}, y_{n}$ in $k^{1 / p}$ are $p$-independent over $k$, we see that $K_{n}$ is modular over $k$ with its listed generators as tensor generators. We have $K_{n-1} \subseteq K_{n}$, and hence $K=\bigcup K_{n}$ is a modular extension of $k_{\text {. }}$ Since we know tensor generators, it is evident that $k K_{n}^{p^{r}} \cap k^{1 / p}$ equals $k$ for $r>n$ and equals $k\left(y_{n}, x_{r}, x_{r+1}, \ldots, x_{n}\right)$ for $r \leq n$. Hence $k K^{p^{r}} \cap k^{1 / p}$ is $k\left(y_{r}, x_{r}, x_{r+1}, x_{r+2}, \ldots\right)$.

To show now $(K / k)^{\omega}=k$ it suffices to show $\bigcap\left(k K^{p^{r}} \cap k^{1 / p}\right)=k$, since an extension with no elements of exponent 1 is trivial. Clearly $k K^{p^{n+1}} \cap k\left(y_{0}, x_{0}, \cdots, x_{n+1}\right)=k\left(y_{n+1}, x_{n+1}\right)$; if we show $k\left(y_{n+1}, x_{n+1}\right) \cap$ $k\left(y_{0}, x_{0}, \cdots, x_{n}\right)=k$ that will suffice, since $k^{1 / p}=\bigcup_{k}\left(y_{0}, x_{0}, \ldots, x_{n}\right)$.

Let $E$ be $k\left(y_{n+1}, x_{n+1}\right) \cap k\left(y_{0}, x_{0}, \ldots, x_{n}\right)$. Considering dimensions over $k$ and noting that the join of the two fields is $k\left(y_{0}, x_{0}, \ldots, x_{n+1}\right)$, we see that either $E=k$ or $|E: k|=p$; and in the latter case the two fields are linearly disjoint. Now $x_{n+1}$ is not in $k\left(y_{0}, x_{0}, \ldots, x_{n}\right)$, so 1 and $x_{n+1}$ are linearly independent over that field. We have the relation $y_{n+1}=$ $\left(x_{n}\right) x_{n+1}+\left(y_{n}\right) 1$ over $k\left(y_{0}, x_{0}, \ldots, x_{n}\right)$, and by linear independence the coefficients are unique. If the two fields are linearly disjoint, there must be such a relation over $E$, and by uniqueness it must be the same relation. Hence $x_{n}, y_{n} \in E$, an impossibility since they are $p$-independent over $k$ and $|E: k| \leq p$. This contradiction proves $(K / k)^{\omega}=k$.

If $K / k$ were a tensor product of simple extensions, then any finite subextension $F$ of $K \cap k^{1 / p}$ would be contained in a finite subextension $F^{\prime}$ of $K \cap k^{1 / p}$ with $K / F^{\prime}$ modular. Thus we can complete the proof as follows. Let $F=k\left(y_{0}, x_{0}\right)$. We will show that if $F \subseteq F^{\prime} \subseteq K \cap k^{1 / p}$ and $K / F^{\prime}$ modular, then $F^{\prime}=K \cap k^{1 / p}$. This we do by proving inductively that $k\left(y_{0}, x_{0}, \cdots, x_{n}\right) \subseteq F^{\prime}$ implies $x_{n+1} \in F^{\prime}$.

By 1.4(a) we have $F^{\prime}$ and $k K^{p^{r}}$ linearly disjoint for all $r$, and hence $F^{\prime}$ is linearly disjoint from $k K^{p^{n+1}} \cap k^{1 / p}=k\left(y_{n+1}, x_{n+1}, x_{n+2}, \cdots\right)$. Call the latter field $T$. Since by induction $y_{0}, x_{0}, \ldots, x_{n}$ are in $F^{\prime}$, we have $y_{n}$ also in $F^{\prime}$. The relation $y_{n}=\left(-x_{n+1}\right) x_{n}+\left(y_{n+1}\right) 1$ expresses $y_{n} \in F$ in terms of $x_{n}, 1 \in F$ over $T$; since $x_{n} \notin T$ the expression is unique. By linear disjointness then the coefficients must be in $T \cap F^{\prime}$, and thus $x_{n+1} \in F^{\prime}$.

This example illustrates why linear disjointness has to enter as a "straightening condition" in many of the earlier proofs. In particular, it shows that the condition " $K / S_{n}$ modular" cannot be dropped from 3.1(iii). For in our example the countability of $K \cap k^{1 / p}$ automatically makes it a 
union of a chain of finite-dimensional intermediate fields $S_{n}$; and each $S_{n}$ has bounded height in $K$, since it is finite and $\bigcap_{k K^{p^{r}}}=k$. Yet $K / k$ is not a tensor product of simple extensions.

Theorem 7.2. Let $m$ be any infinite cardinal. Then there is a modular extension $K / k$ with $|K: k|=m$ having $(K / k)^{\omega}=k$ and $f_{n}\left(K^{\prime} / k\right)=1$ for all finite $n$.

Proof, We first claim that if $F$ is a field of cardinality $m$, then $F^{\boldsymbol{X}_{0}}$ as an $F$-space has dimension at least $m$. (It follows easily that its dimension is precisely $m^{\boldsymbol{X}_{0}}$, but that will not matter.) Suppose indeed that we have a basis of the product containing fewer than $m$ elements; obviously for this to happen $m$ must be uncountable. The entries in the basis vectors then form a set of cardinality less than $m$, and so generate over the prime field a subfield $L$ of cardinality less than $m$. Now an $F$-linear combination of elements in $L^{X_{0}}$ must have all its entries in a finite-dimensional $L$ subspace of $F$. But \#(F)=m>\#(L) certainly implies $|F: L|$ infinite, so we can produce an element in $F^{\boldsymbol{X}_{0}}$ not having all its entries in such a finite-dimensional space.

Now let $F$ be a perfect field of cardinality $m$. Choose $m$ linearly independent elements in $F^{K_{0}}$ starting with the elements $e_{n}$ which are 1 in the $n$th place and 0 elsewhere. Let $V$ be the $F$-space spanned by these $m$ elements. Let $V_{n}$ be the elements in $V$ with first $n$ entries equal to 0 . Then we have a chain of subspaces $V=V_{0} \supset V_{1} \supset V_{2} \supset \ldots$ where $\operatorname{dim} V$ $=m, \operatorname{dim} V_{n} / V_{n+1}=1$ for all $n$, and $\bigcap v_{n}=0$.

Let $k=F(V)$, the fraction field of the free commutative algebra on the vector space; it is a pure transcendental extension of $F$ with transcendence basis = a linear basis of $V=a$-basis of $k$ over $k^{p}$. Let $K_{1}$ be $k\left[V^{1 / p}\right]$, that is, $k\left[\left\{x^{1 / p} \mid x \in V\right\}\right]$; since $F$ is perfect this is the same as adjoining to $k$ the pth roots of the elements in a basis of $V$. Inductively define

$$
K_{n}=K_{n-1}\left[V_{n-1}^{1 / p^{n}}\right]=k\left[e_{0}^{1 / p}, e_{1}^{1 / p^{2}}, \ldots, e_{n-2}^{1 / p^{n-1}}, V_{n-1}^{1 / p^{n}}\right]
$$

Clearly $K_{n} / k$ is modular, and hence $K=\bigcup K_{n}$ is modular over $k_{\text {. Obvi- }}$ ously $|K: k|=m$. We have $k K_{n}^{p^{r}} \cap k^{1 / p}=k\left[V_{r}^{1 / p}\right]$ for $n>r$, and thus $(K / k)^{r} \cap k^{1 / p}=k\left[V_{r}^{1 / p}\right]$. Therefore $e_{r}^{1 / p}$ is a $p$-basis for $(K / k)^{r} \cap k^{1 / p}$ over $(K / k)^{r+1} \cap k^{1 / p}$, and so $f_{r}(K / k)=1$ for all finite $r$.

Finally, $(K / k)^{\omega}=k$ follows easily from 5.7. For it is enough to show $\mathcal{U}\left((K / k)^{\omega} \otimes L\right)$ is trivial, and hence enough to show $\bigcap \mathcal{U}\left((K / k)^{r} \otimes L\right)=0$. But clearly $U(K \otimes L)$ is isomorphic to $V \otimes_{F} L$, with the subspaces isomorphic to $V_{r} \otimes_{F} L$; thus the result follows from $\bigcap v_{r}=0$. 
Corollary 7.3. There are modular extensions $K / k$ which are not forms of group algebras.

Proof. Let $K / k$ be as in the theorem, with $m>2^{K_{0}}$. If $K \otimes_{k} L$ were isomorphic to some $L[G]$, then by $\S 5$ we could conclude $p^{\omega} G=0$ and $f_{n}(G)=1$ for all finite $n$. But for abelian groups this implies [1, p. I-146] that $\#(G) \leq 2^{K_{0}}$.

\section{REF ER ENCES}

1. L. Fuchs, Infinite abelian groups. Vols. I, II, Pure and Appl. Math., vol. 36, Academic Press, New York and London, 1970, 1973. MR 41 \#333.

2. N. Jacobson, Lectures in abstract algebra. Vol. III: Theory of fields and Galois theory, Van Nostrand, Princeton, N. J., 1964. MR 30 \#3087.

3. L. A. Kime, Purely inseparable, modular extensions of unbounded exponent, Trans. Amer. Math. Soc. 176 (1973), 335-349. MR 47 \#192.

4. W. May, Commutative group algebras, Trans. Amer. Math. Soc. 136 (1969), 139-149. MR 38 \#2224.

5. M. E. Sweedler, Structure of inseparable extensions, Ann. of Math. (2) 87 (1968), 401-410; ibid.(2) 89 (1969), 206-207. MR 36 \#6391; 38 \#4451.

6. R. Rasala, Inseparable splitting theory, Trans. Amer. Math. Soc. 162 (1971), 411-448. MR $44 \# 1648$.

DEPARTMENT OF MATHEMATICS, CORNELL UNIVERSITY, ITHACA, NEW YORK 14853

Current address: Department of Mathematics, Pennsylvania State University, University Park, Pennsylvania 16802 